\title{
Gesamtinhaltsverzeichnis Klio Band 62
}

Velizar Velkov (Sofija), Das antike Siedlungsleben in Thrakien . . . . . . .

Georgi Mihailov (Sofija), La ville de Satra à Crète et la tribu thrace des Satres . . . . . . . . . . . . . . . . . . . . . .

Vasmka Gerassimova-Tomova (Sofija), Zur Untersuchung einer antiken Stadt am mittleren Strymon (Struma). Bericht auf dem VII. Internationalen Kongreß über Epigraphie in Constanţa .

Anna Balkanska (Sofija), Tirisis - Tirisa - Akra. Die thrakische und römischbyzantinische Stadt am Kap Kaliakra (Scythia Minor)

Ljoba Ognenova-Marinova (Sofija), Quis autem erat Epimenes? . . . . . . . . 47

Zlatozara Gočeva (Sofija), Prêtres éponymes d'Odessos et de Dionysopolis 49

M ARIA Črčrkova (Sofija), Forschungen in Novae (Moesia Inferior) . . . . . . . . 55

Violeta Božılova (Sofija), Contributions épigraphiques de Novae. . . . . . 67

Lodwika Press-Waldemar Szubert-Tadeusz Sarnowski (Warszawa), Novae in 1977 - West Sector. Results of the Polish Archaeological Expedition . . .

Vasilka Gerassimova-Tomova (Sofija), Wirtschaftliche und religiöse Beziehungen Thrakiens zum Osten . . .

Wolfgang Schindler (Berlin), Allegorie der Iulia Augusti als Iphigenie auf dem Bronze-Krater in Varna . . . . . . . . . . . . . . . . . . . . .

Manfred Oppermann (Halle/S.), Untersuchungen zum Weihrelief in den römischen Provinzen Thracia und Moesia Inferior . . . . . . . . . . . . .

MaRIA KoHLeRT (Berlin), Bemerkungen zu einer römischen Gesichtsmaske aus Varna . . . . . . . . . . . . . . . . . . . . . . . 127

GERDA voN BÜLOw (Berlin), Thrakerdarstellungen in der römischen Kunst . . . 139

IRINA A. Šršova (Leningrad), Zum Übergang von der patriarchalischen zur entwickelten antiken Sklaverei . . . . . . . . . . . . . . . . . . . 157

Konrad H. Krnzl (Peterborough, Ontario), Zur Vor- und Frühgeschichte der attischen Tragödie. Einige historische Überlegungen ～. . . . . . . . . . 177

Virginia Hunter (Downsview, Ontario), Thucydides and the Uses of the Past . 191

Johannes Irmscher (Berlin), Klassische Altertumswissenschaft im „Dritten Reich". Quellen und Forschungsaufgaben . . . . . . . . . . . . . . . 219 
RIgobert GüNTHeR (Leipzig), Bemerkungen zu Váradys „Auflösung des Altertums“

Horst Dieter (Potsdam), „Soziale Konflikte“ in der späten römischen Republik 229

Liselot Huchthadsen (Rostock), Isidorisches bei Isidor von Sevilla

Johannes Irmscher (Berlin), Aus der Arbeit der Fédération Internationale des Associations d'Études Classiques .

Ernst GÜnther Schmidt (Jena), MEGA 2 IV/1. Bemerkungen und Beobachtungen

Jean Fadcounau (Potomac, Maryland), Reflexions sur le déchiffrement des inscriptions cariennes

Peter Karavites (Bridgewater, Massachusetts), The Enduring Mystery of Nicias (Thuc. 2. 85. 5

Rusudan Rzchiladze (Tbilissi), L'Orient dans les œuvres de Xénophon

Paul Todd Makler (Philadelphia, Pennsylvania), New Information on Nutrition in Ancient Greece

Keith R. Bradley (Stanford, California), Sexual Regulations in Wet-Nursing Contracts from Roman Egypt . . . . . . . . . . . . . . . . . . . .

Rhona Beare (Newcastle, New South Wales), Ptolemy's Daimon and RulerCult

Stegbert Hummel (Plohn i. V.), Notizen zu einem etruskischen Stamnos in Altenburg

RONALD T. RIDLEy (Parkville, Victoria), Livy and the concilium plebis

Robert Develin (St. Lucia, Queensland), The Roman Command Structure and Spain 218-190 B. C.

Boris P. SteleckiJ (Leningrad), Zum Gerichtsgesetz des Quintus Servilius Caepio, Konsul des Jahres 106 v. u. Z.

Edward DĄBRowa (Kraków), Le limes anatolien et la frontière caucasienne au temps des Flaviens

Antonino Grillone (Palermo), Pseudo-Hyginus, de metatione castrorum . 389

Jenő Frtz (Székesfehérvár), Die Verwaltung von Pannonia Inferior zur Zeit des Partherkrieges (162-166)

JózeF WoLSkI (Kraków), Le rôle et l'importance des guerres de deux fronts dans la décadence de l'Empire romain

Lucrano Canfora (Bari), Proscrizioni e dissesto sociale nella repubblica Romana

ToRU Yuge (Tokyo), Die römische Kaiseridee. Zur Herrschaftsstruktur des Römischen Reiches

Ramsay MacMullen (New Haven, Connecticut), How Big was the Roman Imperial Army? 
Jean-Claude Richard (Nantes), Les funérailles des empereurs romains aux deux premiers siècles de notre ère. Essai de mise au point . . . . . . . . 461

David M. NovaK (Chicago), Anicianae domus culmen, nobilitatis culmen . . . . 473

Gonzalo Bravo (Salamanca), Para la problemática social de la Roma tardía. Periodización y estructuras . . . . . . . . . . . . . . . . . . . . 495

Georgios Fatouros (Berlin-West), Zur Prokop-Biographie . . . . . . . . . 517

Helene Homeyer (Neu-Esting/München), Zur Synkrisis des Manuel Chrysoloras, einem Vergleich zwischen Rom und Konstantinopel. Ein Beitrag zum italienischen Frühhumanismus . . . . . . . . . . . . . . .

Hermann Srmon (Berlin), Ein Nachtrag zur Ehrung des Johann Jacob Reiske (1716-1774), in: KLIO Band 58/I [1976], $211 \mathrm{ff.} \mathrm{.}$

Detlef Rössler (Berlin), Klassische Archäologie als historische Wissenschaft

IsTVÁN HAHN (Budapest), Die marxistische Kulturgeschichte der Antike . . . . 537

FrITz Jürss (Berlin), Die Entwicklung der religiösen Begriffsbildung. Bemerkungen zu I. Hahn, Götter und Völker ． . . . . . . . . . . . . . . .

Jürgen Dummer (Berlin), Zahlenprobleme in historischen Quellen .... . 561

Wolfgang Schundler (Berlin), Ein neuattisches Werk des 4. Jahrhunderts v. u. Z.?

Burkhard BötTGER (Berlin), Conimbriga - vom keltischen oppidum zum flavischen municipium . . .. . . . . . . . . . . . . . . . . . . . . . .

Hans Krummex (Berlin), Zum Firmalampen-Katalog des Archäologischen Museums in Aquileia . . . . . . . . . . . . . . . . . . . . .

Pierre Briant (Toulouse), Remarques sur „Wirtschaft und Gesellschaft im Seleukidenreich" de H. Kreißig . . . . . . . . . . . . . . . . . .

Barbara Zuchold (Jena), Die sogenannte römische Revolution und Alfred $\mathrm{Heuß} \mathrm{.} \mathrm{.} \mathrm{.} \mathrm{.} \mathrm{.} \mathrm{.} \mathrm{.} \mathrm{.} \mathrm{.} \mathrm{.} \mathrm{.} \mathrm{.} \mathrm{.} \mathrm{.} \mathrm{.} \mathrm{.} \mathrm{.} \mathrm{.} \mathrm{.} \mathrm{.} \mathrm{.} \mathrm{.} \mathrm{.} \mathrm{.} \mathrm{.} \mathrm{.} 583$

Kraus-Peter Johne (Berlin), Nordafrika im Widerstand gegen Rom . . . . . 593

JürGEN Dummer (Berlin), Zwei neue Bände der Eusebius-Ausgabe in den „Griechischen Christlichen Schriftstellern“ . . . . . . . . . . . . . . . . . 597

Helga Köpstern (Berlin), Zu zwei Neuerscheinungen der Byzantinistik . . . 603 Hans Krummey (Berlin), Zu L'Année épigraphique 1973 . . . . . . . . . . 611

Hans Krummrey (Berlin), Zu zwei neuen Bänden der Bibliographie zur griechischen Sprache . . . . . . . . . . . . . . . . . . . 615

Liselot Huchthausen (Rostock), Alter Wein aus neuen Schläuchen. Zur Nachdruckserie „Juridica“ . . . . . . . . . . . . . . . . . . . . . . . 619

Liselot Huchthausen (Rostock), Mißbrauchter Cicero . . . . . . . . . . 623

Friedhelm Winkflmann (Berlin), Byzanz zur Zeit der Kaiserin Eirene . . . 625

Bis zum 15. 6. 1979 bei der Redaktion der Klio eingesandte Literatur . . . 633 
